\title{
Dominique Mahieu-Caputo
}

\section{F. Molénat \\ (C) Springer-Verlag France 2010}

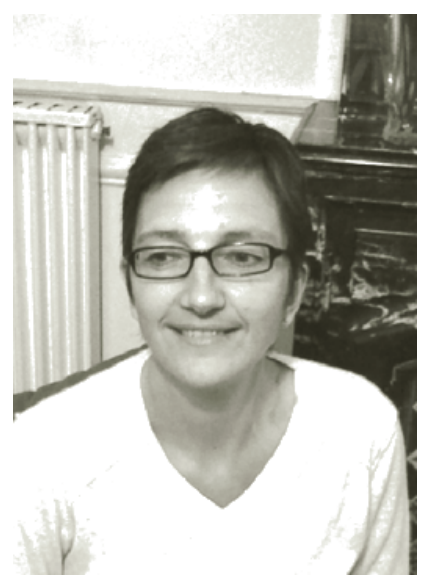

Il m'est difficile d'évoquer la disparition d'une amie, personnalité hors du commun, tant sa présence lumineuse faite d'intelligence et de générosité nous a tous marqués. Le parcours professionnel de Dominique Mahieu-Caputo témoigne d'une énergie et d'une ouverture d'esprit exceptionnelles. Ses qualités humaines et son appétit vers le beau, le bien, le neuf ont fait de sa trajectoire suspendue en plein élan un modèle de rayonnement qui n'est pas près de s'éteindre.

Nous nous étions rencontrées lors d'une expertise collective de l'Inserm sur le handicap d'origine périnatale. Légèrement en retard, elle nous dit gaiement sortir d'une garde au cours de laquelle elle avait accompagné la naissance de huit enfants de mères en parcours de migration : « grossesses non suivies ». Sa bonne humeur me frappa, et ses propos dénués de tout élément négatif, bien au contraire. Une part de son énergie venait manifestement de cette participation à la vie, et j'aurai l'occasion maintes fois d'apprécier la vitalité qui se dégageait d'elle et qu'elle savait transmettre par des voies intellectuelles ou amicales.

Très discrète sur son propre chemin, elle me laissa découvrir, par la littérature, ses travaux novateurs sur le syndrome transfuseur-transfusé puis sur les cellules souches hépatiques et, par les amis, son remarquable parcours universitaire. Sa capacité étonnante à s'affronter à de nouveaux champs d'intérêt est citée par tous.

Le hasard des colloques nous rapprocha à plusieurs reprises. Ses propos sur l'annonce d'une anomalie foetale m'ont impressionnée, tant elle savait intégrer à leur juste place les multiples éléments émotionnels en jeu pour la femme, son conjoint, le couple, les professionnels, avec la même rigueur qu'à l'exposé du déroulement technique. Inutile de parler de « psychologie », il s'agissait bien d'une médecine périnatale 《 intégrée ».

Pourtant, à l'annonce du Plan périnatalité 2005-2007, il fallut créer une société de psychologie périnatale destinée à baliser le chantier ouvert par l'objectif de sécurité émotionnelle des femmes enceintes. Il était trop tôt pour que les tra- vaux s'intègrent dans la réflexion médicale stricto sensu, trop tôt pour parler de démarche « écologique ». L'importance de l'environnement humain de la naissance, la nécessité d'accueillir les ressentis des femmes enceintes dans un bon suivi de grossesse s'imposaient à elle. Elle s'est donc engagée dans les travaux et se mobilisa fortement pour la création d'un diplôme interuniversitaire de "psychopérinatalité ». Le programme s'est élaboré avec elle et avec les collègues d'obstétrique, de pédiatrie, de pédopsychiatrie intéressés à transmettre des outils pertinents aux praticiens de la périnatalité : il s'agissait d'optimiser les conditions d'accueil des parents et des enfants et de prévenir ainsi une série de troubles parfaitement évitables. Elle s'impliqua fortement par sa présence physique tout au long de la première année.

Passionnée par ces aspects, elle me sollicita pour réfléchir avec son équipe, le Réseau périnatal, les partenaires qu'elle avait su mobiliser pour le suivi des femmes enceintes dans un contexte de précarité. Même si la maladie l'empêchait souvent d'être là, on ne pouvait que répondre « présent » devant cette énergie communicative. La participation nombreuse de praticiens du secteur libéral, en particulier de médecins généralistes, signait la réussite de ses projets.

Elle s'est engagée dans la Revue de médecine périnatale nouvellement créée, et nous fit ce beau cadeau aux dernières Journées de la société, à Angers, d'un remarquable exposé sur " grossesse et précarité ». Elle avait tenu à venir, tout en se battant contre les complications de la maladie, et nous ne sommes pas prêts d'oublier sa présence lumineuse à la table ronde et en soirée, alors qu'elle apprenait de mauvaises nouvelles, sans rien en dire. Jusqu'aux derniers jours, nous avons travaillé sur des projets qui - disait-elle - lui donnaient de la force.

Consciente que la rencontre avec Dominique MahieuCaputo fut une chance pour moi-même et beaucoup d'entre nous, je pense à ses amies proches qui ont magnifiquement témoigné de leur belle relation. Je tiens à remercier Véronique Mirlesse et Sylvie Epelboin qui m'ont tout particulièrement parlé de leur longue complicité, et dont la présence chaleureuse atténue notre tristesse. Dominique évoquait souvent l'énergie qu'elle puisait dans ces amitiés.

Mais durant ces années, elle parla aussi de ses enfants, d'Héloïse la dernière venue, du soutien indéfectible de son mari, et c'est vers eux que vont toutes mes pensées, sachant combien leur présence allégeait le poids des événements, et ressentant leur peine. 